\title{
基于网络的 3DGIS 可视化研究
}

\author{
张立强 (1) ${ }^{*}$, 过志峰 (2), 康志忠 ${ }^{3}$, 张立新 ${ }^{1}$, 杨玲 (1) \\ (1) 北京师范大学地遥学院遥感科学国家重点实验室, 北京 100875 ; \\ (2) 中国科学院遥感应用研究所, 北京 100875 ; \\ (3)中国地质大学(北京)土地科学技术学院, 北京 100083 \\ *通信作者 E-mail: zhanglq@bnu.edu.cn
}

收稿日期: 2008-05-24；接受日期: 2009-05-30

国家高技术研究发展计划 (批准号: 2006AA12Z220, 2006AA12Z114)、国家重点基础研究发展计划（批准号: 2007CB714403）和教育部新 世纪优秀人才计划 (批准号: NCET-07-0099) 资助项目

摘要自适应可视化大规模复杂的空间数据已成为三维地理信息系统 (3DGIS) 的 研究热点. 为了从服务器传输可视化信息到客户端, 文中提出了结点一层数据模型来 组织三维场景, 该三维场景提供了有效的数据管理方式, 包括空间索引和渐进传输算 法. 服务器根据顶点和三角形数目, 对场景元素不是提供几个预先计算的细节层次, 而 是连续分辨率的表达. 客户端根据硬件的性能对复杂三维场景自适应多分辨率可视化, 满足多用户并发访问的要求. 实验证明, 文中的方法能有效地解决大规模复杂空间数 据模型的快速可视化.

\section{关键词}

数据模型

渐进传输

空间索引

自适应可视化

\section{0 引言}

近几年, 随着传感器和数据采集技术的不断发 展, 大范围、高分辨率影像和数字高程模型的廉价获 取成为可能, 空间数据量呈几何级数增长. 随着几 何对象数量的不断增加和几何表达精度的不断提高, 用于描述几何对象的数据量也变得越来越多, 再加上 从其他方法 (如测量方法) 获取的 GIS 数据, 一个系 统的数据总量往往达到几百兆字节甚至 TB 数量级, 而且这些数据的格式也不尽相同. 从而使得传输和 可视化这些空间数据需要的计算机内存和资源远远 超出了计算机设备的承受能力. 在保证场景真实感 的前提下, 快速实现多源海量数据的可视化成为当 前空间信息领域的一个挑战.

为了在网络环境下, 提高大数据量空间数据 (数 字高程模型、影像和建筑实体) 的可视化速度, 本文 提出了结点一层数据模型来组织三维空间场景, 服 务器根据顶点和三角形数目, 通过空间索引和渐进传
输算法对场景元素进行连续分辨率的描述, 客户端根 据本身硬件的性能自适应对复杂三维场景多分辨率 可视化. 与传统方法相比, 本文的方法在网络传输速 率较低的情况下, 能够保持较快的可视化速度, 能满 足多用户并发访问的要求.

\section{1 相关研究}

国内外很多科研机构在大数据量的 3DGIS 方面 做了大量工作. 具体包括:

\section{1 空间数据模型和网络传输}

GIS 中的空间数据模型是通过一定的数学模型 对现实世界的几何对象进行抽象、归类及简化的描 述和表达, 分为二维数据模型和三维数据模型. 二维 数据模型发展比较成熟, 三维数据模型正处在不断发 展和完善之中. Losa 等 ${ }^{[1]}$ 保留了二维对象的拓扑关 系, 在扩展方向弧的基础上描述了 3DGIS 对象模型. Gold 等 ${ }^{[2]}$ 提出用 Voronoi 图和双重 Delaunay 三角 网构建地球椭球的拓扑关系, 同时在三维空间中采用 
方向余弦作为坐标系统基准来构建椭球表面的实体, 该方法能实现对地球椭球及其表面实体连续无缝的 表达, 但是建立拓扑关系的数据结构复杂, 计算量大. Coors $^{[3]}$ 提出了基于查询的空间数据模型 UDM 来表 达城市建筑模型之间的邻接关系, 通过利用凸平面描 述面状和体状实体的几何特性, 但 UDM 模型不支持 一维点状地物, 而且随着实体复杂度的增加, 所需凸 平面的数目迅速上升.

在国内, 龚建雅等 ${ }^{[4]}$ 提出了矢量栅格集成的混 合数据模型, 并在数据模型中定义了结点、弧段、断 面、体元等空间地物和一个数据结构. 李清泉等 ${ }^{[5]}$ 提出了基于 TEN+Octree 的空间三维实体的混合数 据模型. 杨必胜 ${ }^{[6]}$ 将面向对象的数据模型用于表达 和存储三维几何目标. 这些数据模型对特定实体的 建模方面具有一定的优势, 但是难以实现空间实体 的细节层次模型的表达. 到

目前的网络带宽和传输速率难以适应大规模空 间数据快速传输的需要, 若将整个模型完全下载到本 地再渲染, 等待时间长. 因此加快空间数据传输的有 效途径是降低网络传输负担, 而且场景的描述要适应 客户端的实际处理能力.

允许空间数据远程下载的传统方法是对模型简 化后渐进传输. Teler 等人 ${ }^{[7]}$ 提出了服务器端只渲 染最低层次的元素, 将处理结果以图像的形式返回客 户端, 而其他的细节层次 (LOD, level of detail) 作为 三维模型传到客户端后再可视化. 该过程类似于视 频流方法, 最低一层 LOD 的渲染时间加上栅格图像 传输时间要少于直接传输三维模型的时间, 但大量元 素和客户机同时运行时, 即使较低细节层次的可视化 过程也会变得非常复杂, 服务器的负载大. Kim 等 ${ }^{[8]}$ 提出渐进面的视相关流技术. 服务器将基本面和顶 点的分级结构传到客户端, 接下去, 根据客户端发出 的选择性简化数据请求, 服务器把剩余数据渐进传输 到客户端. Danovaro 等 ${ }^{[9]}$ 实现了一个客户端/服务器 原型系统, 在客户端完成 LOD 查询而不必接收和存 储整个 LOD 模型, 服务器存储整个数据模型, 当接 收到客户端的请求时, 服务器通过空间索引检索出合 适的数据, 进行简化后传到客户端, 从而在一定程度 上保持了负载平衡.

另一种方法是基于流的渐进传输技术, 即服务器
先传输较低分辨率的模型, 然后通过一系列的边界分 割算法后连续的传输、补充和细化整个模型 ${ }^{[10 ~ 12] . ~}$ 因为空间模型的结构和其连接关系要存储在内存中, 几何模型若有上百万个三角形组成, 超过了 PC 机主 存的大小, 该方法的传输效率会显著降低. 最近出现 了称之为外存流的技术, 其中之一是基于点采样的 几何模型 Qsplat 算法 ${ }^{[13,14]}$, 它逐级的传输实体并能 做视相关简化. Rusinkiewicz ${ }^{[14]}$ 等人提出 Streaming QSplat 的概念, 将模型序列化为线性结构, 在网络中 只传输点模型, 通过遍历层次树来完成整个模型的绘 制, 但是在平坦的区域, 点采样要比多边形需要更多 的数据, 从而带来大量的觉余数据.

\section{2 快速可视化技术}

为了加快大数据量空间数据的可视化速度, 实现 实时交互操作, 通常大规模场景的实时渲染是通过模 型的简化来实现的, 即用较粗粘的格网代替高分辨率 的细分格网来减少待渲染的多边形数目. 不足之处 在于, 运行期间不同层次的 LOD 模块切换时会出现 裂缝和突跳现象.

当前图形硬件每秒钟能够渲染几十万甚至上百 万个三角形, 但是大多数可视化方法达不到这个性 能. 从多分辨率模型中提取合适的细节层次并将其 传输到图形加速卡是三维可视化的主要瓶颈, 受浮 点数值描述范围所限, 高精度的几何模型在可视化 过程中会伴随着精度损失, 引起场景不连续性. 根据 图形硬件的特性, 相继提出了适应图形硬件的算法, 如 Geometrical mipmap (Geomipmap) ${ }^{[15]}$ 、Chunked LOD 以及 Geometrical Clipmap ${ }^{[16]}$. 这些算法通过 在图形卡内存上建立高速缓冲, 极大提高了绘制速 度, 并且绘制效果得到改善, 节约了大量 CPU 计算 时间, 使得 CPU 更好地进行数据调度等其他方面的 运算, 但是在绘制复杂城市场景时, 由于判断空间实 体的拓扑关系, 仍然需要较大的计算量.

\section{3 三维地理可视化平台}

近年来, 可视化技术有了长足的发展, 很多图像 处理软件和 GIS 软件增加了三维可视化的功能. 但 是对复杂实体的建模主要依靠 $\mathrm{CAD}$ 或三维造型软 件来完成, $\mathrm{CAD}$ 图形元素是由单一边构成, 缺乏对空 间实体管理和操作能力, 在一定程度上增加了三维模 型创建的难度和费用, 导致较差的模型视觉效果, 因 
此 CAD 系统无法满足某些应用领域的研究需要. 另 一方面, 空间分析基本上还是局限于 $2 \mathrm{D}$ 或 $2.5 \mathrm{D}$ 分 析功能.

在国外, Google 公司在 Keyhole 公司开发的 EarthView 基础上推出了桌面工具 Google Earth. 它 是迄今为止最完善的影像服务平台, 能够实现海量影 像、DEM 和矢量数据的网络传输、可视化等功能, 但 是其空间分析能力较弱. 从 90 年代中期开始, 美国 Skyline 公司开发了 Skyline TerraSuite 三维 GIS 软 件, 在交互式 3D 空间中, TerraSuite 能够无缝集成航 空影像、卫星数据、数字高程模型和其他 GIS 数据, 在快速数据融合、支持大型数据库和实时信息流通 讯技术等方面具有很好的优势. NASA World Wind 是美国航空航天管理局 (NASA, national aeronautics and space administration) 推出的开源三维虚拟地球 可视化系统, 它将 NASA、美国地质调查局 (USGS, United States geological survey) 以及其他网络地图 服务商提供的图像通过一个三维地球模型展现, 该 系统的不足之处在于第一次运行该系统必须先下载 $52 \mathrm{MB}$ 的文件.

国内在三维可视化领域发展迅猛, 灵图软件技术 公司的 VRMap、适普公司的 IMAGIS 以及武汉吉奥 公司的 CCGIS 都带有处理复杂空间模型的功能. 在 可视化的效果上, VRMap 占一定的优势, 并在诸如水 利、规划、环保等领域取得了较好的应用. IMAGIS 是以 IDL 为开发语言的基础上形成的, 因而具有较 强的三维模型造型功能, 但是在大场景的汶览速度上 受到一定的限制.

\section{2 面向可视化的数据模型}

\section{1 数据模型}

本文提出结点一层的数据模型 NVDM (nodeview data model) 组织三维场景的空间数据. NVDM
可以用图 1 描述.

三维场景的元素可以包含多个信息以描述不同 的实体类型, 如面的顶点、纹理坐标值和颜色. 顶点 和纹理坐标属于几何信息, 而颜色和纹理描述的是影 像信息. 另一个重要信息是顶点之间的连接关系, 即 元素的拓扑关系.

NVDM 根据空间实体的类型特征, 把空间对象 划分成若干类, 每个类依据各自的特征定义数学模 型, 形成结点. 任意结点可以是另外结点的子结点, 每个结点都有各自的参考系统、空间信息以及属性 特征. 通常, 这些结点之间存在复杂的空间关系, 因 此, 引入 “层” 概念来组织这些结点, 每层的结点与结 点视图显示的对象有关 (图 2). 元素之间的可视化特 征借助于元素图来描述, 元素图的每一个结点代表着 具体的数据类型, 例如, 顶点数组结点指向元素的顶 点, 纹理坐标的属性用顶点或面来定义, 说明不同的 结点类型. 每个元素图组成一层. 每个结点对应于数 据库中的一条记录, 层跟数据库的表进行绑定. 通过 这种方式, 实现几何对象与其对应的属性之间交互式 操作, 例如属性查询或空间地址的查找等.

为了避免三维场景元素间的数据冗余, 数据存储 在几个线性表中, 元素图的结点是这些线性表入口的 引用. 根据元素图结点类型, 系统提供合适的线性表 类型, 如几何线性表存储顶点或纹理坐标; 拓扑线性 表存储拓扑信息. 线性表以紧凑高效的方式存储了 三维场景的全部可视化信息.

下面论述 NVDM 模型中 DEM、纹理、点、线、 面和体及其对应属性信息的存储结构 ${ }^{[17]}$.

DEM、纹理数据的组织结构如表 1 所示.

在结点表 Nodes (表 2) 中存储了每个顶点的三 维坐标信息.

线状实体存放在线表 Lines (表 3) 中, ID 记 录了该实体的序号, Node_Num 记录线结点的个数, Node_Array 说明组成线的结点编号.

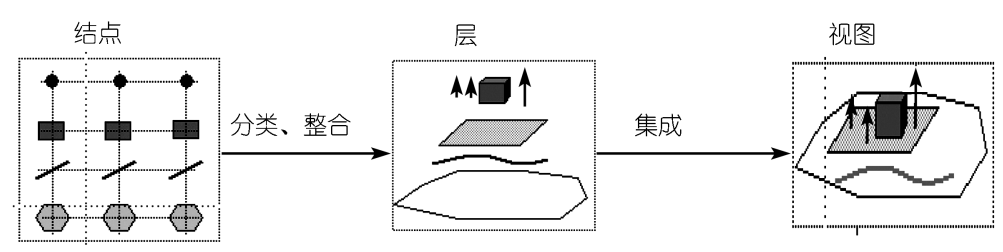

图 1 结点一层一视图数据模型 


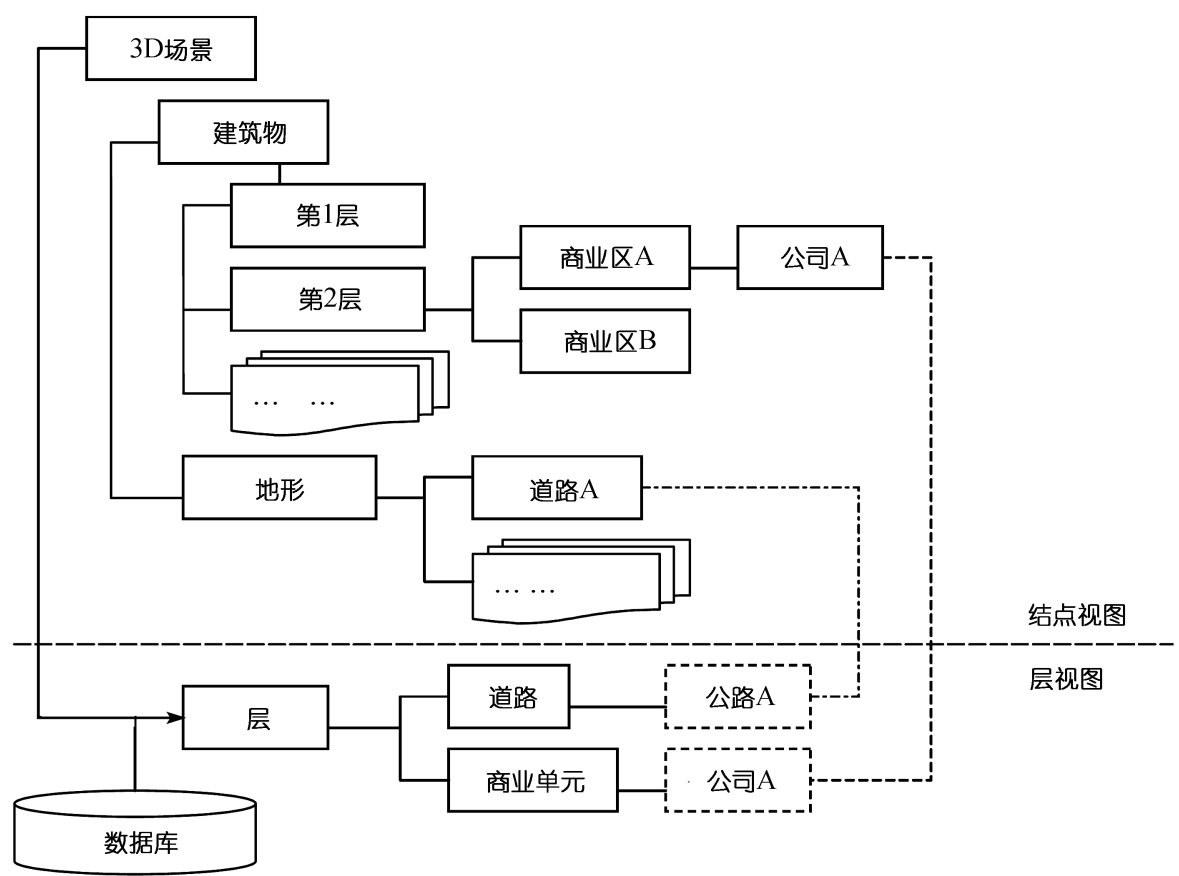

图 2 结点一层的关系

表 1 DEM 纹理数据的组织结构

\begin{tabular}{ccccccccccc}
\hline ID & Level & Name & Type & Cols & Rows & $X_{\min }$ & $Y_{\min }$ & $X_{\max }$ & $Y_{\max }$ & Data \\
\hline 0 & 1 & $\mathrm{SD}$ & 1 & 500 & 750 & 111.40 & 30.77 & 111.46 & 30.81 & Binary \\
\hline
\end{tabular}

\section{表 2 Nodes}

\begin{tabular}{llll}
\hline ID & Cor_X & Cor_Y & Cor_Z \\
\hline$N_{0}$ & 356.78 & 457.23 & 274.21 \\
$N_{1}$ & 409.59 & 457.23 & 274.22 \\
\hline
\end{tabular}

\section{表 3 Lines}

\begin{tabular}{ccc}
\hline ID & Node_Num & Node_ID \\
\hline$L_{1}$ & 3 & $N_{0}, N_{1}, N_{2}$ \\
\hline
\end{tabular}

\section{表 4 Faces}

\begin{tabular}{ccc}
\hline ID & Node_Num & Node_ID \\
\hline$F_{0}$ & 4 & $N_{0}, N_{1}, N_{2}, N_{3}$ \\
$F_{1}$ & 4 & $N_{4}, N_{5}, N_{6}, N_{7}$ \\
\hline
\end{tabular}

面状实体存放在面表 Faces（表 4) 中，ID 记 录了该实体的序号, Node_Num 记录面结点的个数, Node_ID 说明组成面的结点编号.

三维模型由三个表组成: Bodies(表 5)、Floors(表 6) 和 Roofs(表 7). Bodies 主要记录了该模型的墙 面数目、屋顶类型、最小包围范围以及属性, 其中, Floors 说明 Bodies 中墙面的数目, RoofShp 是屋顶 类型, $x_{\min }, y_{\min }, x_{\max }, y_{\max }$ 是模型的最小边框坐标.

墙面 Floors 表中, 一个面可能是两个相邻实体 的公共部分, 因此增加 Floor_left 和 Floor_right 两个 指针. 对独立的实体来说, Floor_left 和 Floor_right 两 者之一指向 Bodies 中的 ID, 另一个为空, 用 -1 表 示. Building_ID 是建筑的编号, Floor_left 是左侧相

表 5 Bodies (3D-Geodata)

\begin{tabular}{cccccccc}
\hline ID & Floors & RoofShp & $x_{\min }$ & $y_{\min }$ & $x_{\max }$ & $y_{\max }$ & Attribute \\
\hline$B_{0}$ & 3 & 0 & 356.78 & 457.23 & 409.59 & 515.83 & Offices \\
$B_{1}$ & 6 & -1 & 162.13 & 213.48 & 209.51 & 252.74 & Residential \\
\hline
\end{tabular}




\begin{tabular}{cccccc}
\hline ID & Building_ID & Floor_left & Floor_right & Face_ID & Attribute \\
\hline$D_{1}$ & $B_{1}$ & $D_{2}$ & -1 & $F_{0}, F_{1}, F_{2}, F_{3}$ & Shop \\
$D_{2}$ & $B_{1}$ & $D_{3}$ & $D_{1}$ & $F_{4}, F_{5}, F_{6}, F_{7}$ & Company \\
$D_{3}$ & $B_{1}$ & -1 & $D_{2}$ & $F_{8}, F_{9}, F_{10}, F_{11}$ & Company \\
\hline
\end{tabular}

邻的墙面, Floor_right 是右侧相邻的墙面, Face_ID 说 明每个墙面由哪些三角形面组成, 三角形面存储在 Faces 表中, Attribute 是该墙面的属性信息.

在 Roofs 表中, 若建筑的房顶形状是金字塔, $\operatorname{RoofShp}=0$, 否则, RoofShp $=-1$.

\section{表 7 Roofs}

\begin{tabular}{cccc}
\hline ID & Building_ID & Face_ID & Attribute \\
\hline 0 & $B_{0}$ & $F_{12}, F_{13}, F_{14}$ & Pyramidal \\
\hline
\end{tabular}

3D-Geodata 结构的不足在于三维实体的正面结 构复杂时 (墙上有窗户), 需要绘制大量的三角形, 但 利用纹理贴图, 将复杂表面简化为简单面来处理, 便 于改善建模的效率.

\section{2 纹理的绘制}

建筑物可看成是一组闭合的由三角形面片构成 的实体, 每个面都附有各自的纹理. 由 3.1 小节, 建 筑物有唯一的 ID 标识, 其上每个多边形也有确定的 标识 (称作子 ID). 如果纹理标识符和建筑物的相同, 则表示纹理归附于该建筑物的表面. 接下去, 搜索与 建筑物的面有相同标识符的纹理子 ID, 如果找到, 绘 制纹理. 重复这一过程, 直到所有的面被映射纹理.

\section{3 自适应可视化}

\section{1 客户端/服务器}

系统启动时, 客户端通过网络与服务器建立了联 系, 配置文件下载到客户端, 通知管理器空间数据分 布的具体位置. 配置文件包含数据类型、大小、所在 的位置以及空间范围等信息, 它放在一组 Hash 表中, 用来管理放在每台服务器上待可视化的多源空间数 据. 客户端可视化过程中, 如果数据不在客户端, 系 统发送请求到服务器, 获取落在视景体中多分辨率模 型信息. 服务器对请求做出响应, 通过 P-树 [12] 空间 索引查找需要的数据. 客户最初接收到服务器发出 的较粗粘数据, 通过跟踪可视化过程, 识别当前可视
化的部分场景. 如果需要, 用户可以在不超过几何近 似值误差 (阈值) 的前提下, 请求服务器发送更细节 的数据, 根据客户端的视点位置和视点方向, 提供可 视化区域更高精度的压缩位流, 并传输到客户端.

依据空间实体到客户端视点距离, 每个被选择的 元素有一个优先级. 一旦视点改变, 元素优先权也随 之改变, 并且可能要求额外的数据. 当客户端有新的 请求, 它预先计算索引后, 发送消息到服务器获取预 先没有存储的模型结点. 为了避免不必要数据的传 输, 客户端从服务器上接收到空间数据时, 它做部分 简单计算, 搭贝一部分数据模型, 客户端的简化算法 修改场景描述, 建立元素图形 (图 3), 该图形包含渐 进入口, 依连续的顺序存储数据 ${ }^{[18]}$. 如果客户端不 能存储整个细节层次模型, 就删除当前不用的数据. 若后续的操作需要这部分数据, 它只简单向服务器 发送一个请求, 因为它不需要记录每个连接点的状 态, 这样服务器非常容易处理大量的客户端连接, 支 持多用户并发访问.

\section{2 数据的传输与可视化}

渐进传输算法遍历给定场景的所有结点元素, 元 素图形内带有拓扑信息的结点必须是索引三角形结 点集, 系统能实时添加新元素图或修改存在的元素图 及其信息, 将当前元素图访问结点引用的链表入口存 入内存. 每次带有拓扑信息的结点到达后, 简化算法 开始运行.

进行每一步简化时, 一个顶点和两个三角形将从 由元素图中当前顶点数组结点和当前索引三角形集 结点表示的面上删除. 为了决定顶点删除的次序, 根 据数据块视觉的重要性, 采取从精到粗的顺序绘制 数据模型. 视觉最重要的数据块, 优先级最高, 最先 绘制, 细节层次也最高. 随着数据块视觉重要性的大 小, 依次绘制整个视景体内的数据. 数据块视觉重要 性 $p_{t}$ 可由 (1) 式计算获得. $p_{t}$ 值最小的顶点首先删 
除, 接着重新计算剩余顶点的误差值, 重复前面的过 程, 直至大于规定的阈值为止.

$$
p_{t}=\alpha \times \frac{\text { max_dist }- \text { dist }_{t}}{\text { sum_dist }}+\beta \times \frac{\text { height }_{t}}{\text { sum_height }_{\text {sum }}} .
$$

对数据块 $t$ 来说, dist $_{t}$ 是数据块的中心到视 点的距离. height $t_{t}$ 是数据块 $t$ 最小包围盒的高度. Max_dist 是所有可见据块中 dist $_{t}$ 的最大值; sum_ dist 和 sum_ height 分别是 dist $_{t}$ 和 height $t$ 累加和; 权 $\alpha, \beta$ 满足 $\alpha+\beta=1$, 为了折衷数据块的距离和高 度, 通常取 $\alpha=\beta=0.5$. 所有可见数据块的视觉重 要值 $\operatorname{imp}_{t}$ 要进行归一化, 这样 $\sum_{t} \mathrm{imp}_{t}=1^{[19]}$.

因为渐进数据反映了删除顺序, 原始面通过简 化过程的逆操作在客户端上重建. 顶点和三角形以 服务器相反的删除顺序插入到基本面上 (图 4). 基于 此, 服务器能够快速的调整数据以适应客户端的运行 能力. 考虑到客户端的能力, 服务器确定应传到客户 端的三角形数目 $n_{t}$ 及顶点的数目 $n_{v}$. 因为服务器总 是传输基本面 $M^{0}, n_{v}$ 和 $n_{t}$ 必须大于基本面相应的 $n_{v}^{o}$ 和 $n_{t}^{o}$. 通常, $M^{0}$ 只含有几个顶点和三角形, 因此 能在弱客户端上渲染. $M^{0}$ 数据传输以后, 服务器传 输顶点数组的前 $n_{v}-n_{v}^{o}$ 个人口. 因为渐进传输, 服 务器不需将所有信息作为数据包传到客户端, 而是分 步进行. 为了减少内存的消耗, 构建三维场景的结点 采用算术编码压缩, 先传输可见子结点中较粗的模 型, 并将其保留在客户机内存中, 渐进可视化更高层 次的场景. 如果遍历停止, 就用较粗细节层次模型渲 染 (图 5). 在遍历 P-树结构搜索当前待渲染结点的 过程中, 将流和预取过程分为两个异步线程进行. 在 交互的过程中必需的数据放在缓存中, 每个细节层次 中保留数据块的缓存, 如果立即使用, 就存储在内存 缓存中, 否则, 放在磁盘缓存中.

\section{3 空间索引}

随着视点和视线不断移近城区, 场景会出现三 维建筑等实体. 空间索引保证靠近视点或用户最感 兴趣的实体先传输, 次要目标后传输和渲染. 在此情 况下, 本文提出一种 P-tree 的空间索引结构, 它在传

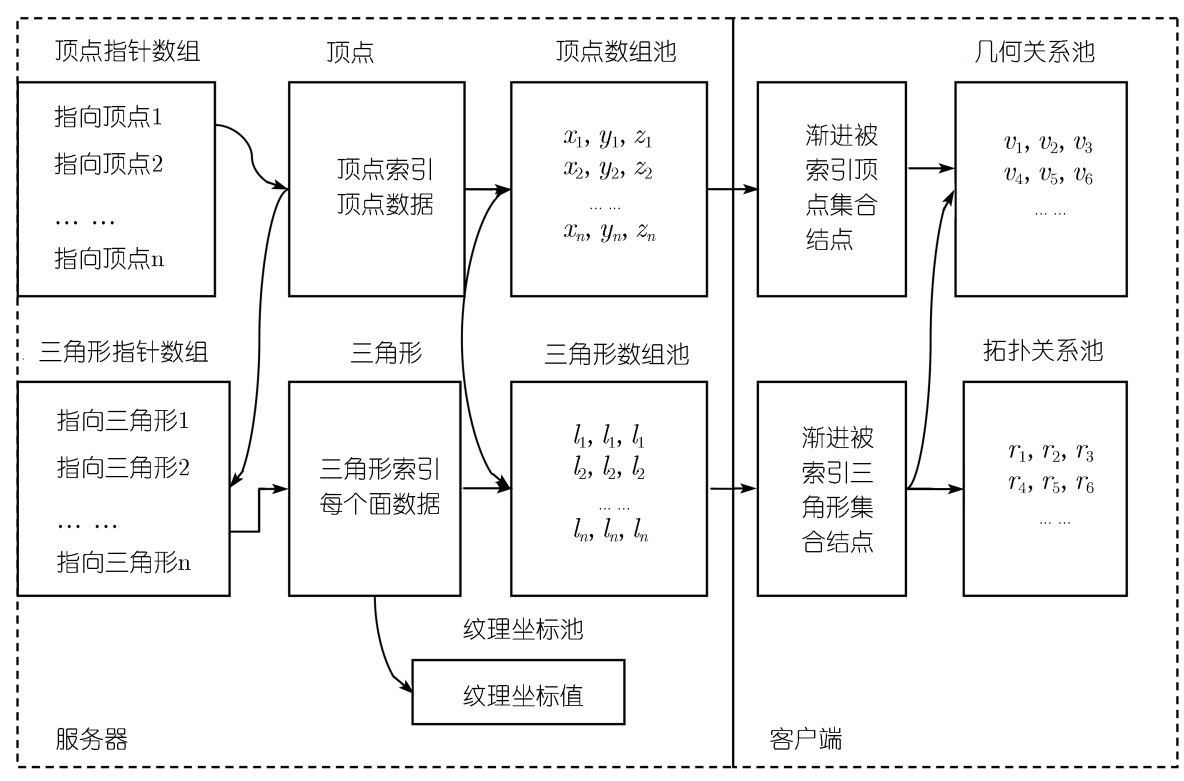

图 3 空间数据的简化与渐进传输

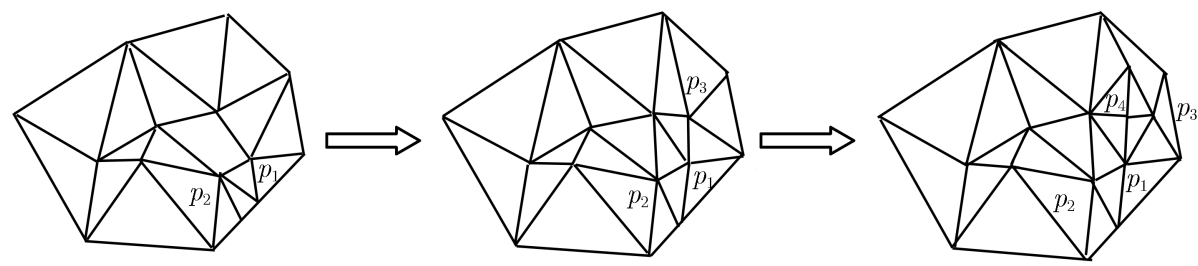

图 4 面的细化过程 
统 $\mathrm{R}$ 树基础上, 增加了一个元素 $g(e)$ 来存储地理空 间信息. 如果第 $L$ 层次的结点 $n$ 是一个内部结点, $g(e)$ 表示在该分支中所有实体集合. 返回的集合在 包围盒精度高于阈值时, 就合并 P-tree 的内部结点, 否则遍历 P-tree 的下一层. 具体参见文献 $[3,12]$.

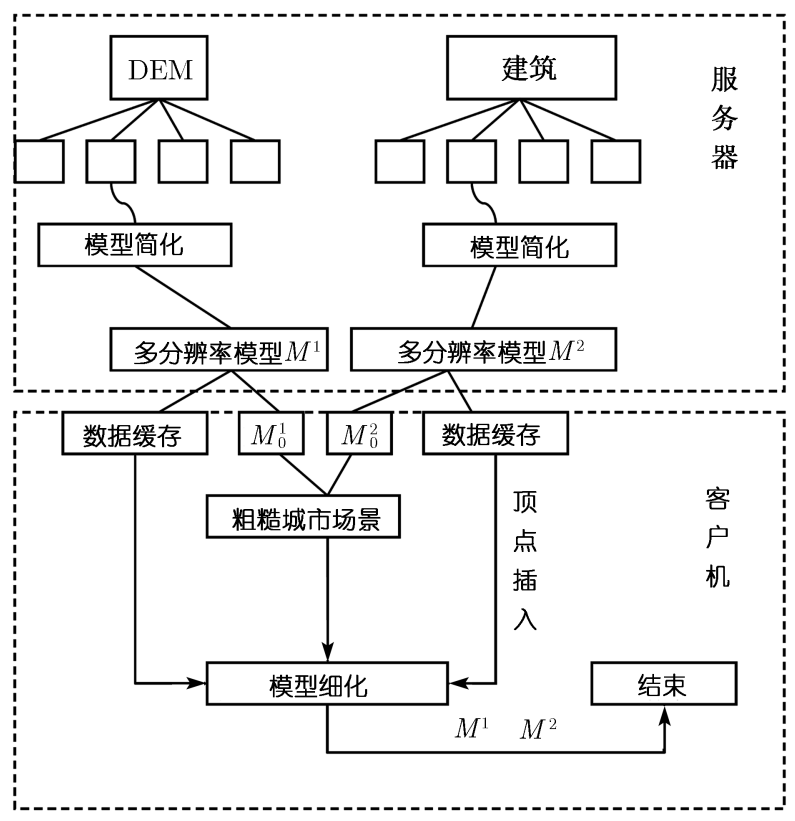

图 5 城市场景的网络传输与可视化

\section{4 视域裁剪和多线程技术}

为了减少三角形绘制的数目, 隐藏面需要被剔 除. 因为只有落在视锥体中的数据是可见的, 如果 结点完全在观察者的视野之外, 它的子结点也要被 删除. 把未剔除的结点屏幕空间误差与阈值误差 比较, 如果前者小于阈值, 则跳过子结点并渲染. 结 点不断进行递归运算直到屏幕空间误差在可接受的 范围内或者被访问结点的最小外接矩形在视雉体之 外.

除了数据更新和顶点索引结构改变需要 $\mathrm{CPU}$ 重 新计算外, 从存储单元中装载新数据到主存储器, 然 后传输到显卡上也需要花费一定的时间 ${ }^{[20]}$. 针对这 一问题, 我们采用多线程技术处理基于视点变化的 空间数据, 多个线程在系统后端运行, 并与外部程序 通信. 主线程负责顶点数组和纹理坐标的更新, 索引 数组的实时运算以及场景渲染. 输入〉输出线程依据 视点的改变动态计算可视区域, 保证另一条线程预 先获取当前层的数据. 因为读取磁盘数据是整个系
统最慢的环节, 而新的几何表面信息又需要从服务 器下载, 若减少反应时间, 就要使预取算法和线程渲 染同时进行. 视点越远, 优先权越大, 这样数据被预 取, 使较为粗粘的层被细化. 如果层数据更新的速度 不及视点移动的速度, 父层连同其子层不能被实时渲 染, 父层就更新为较精细的层次并被渲染. 由于算法 的实现是基于 GPU 的, 能节省更多的 CPU 资源并 可以实现线程的及时调度.

\section{4 实验分析}

在网络环境下对本文的算法进行验证. 服务器 配置是 P4 $2.8 \mathrm{GHz}$ 处理器, 1G\&NVIDIAFX3400 的 3D 显卡, 操作环境为 IIS/Windows XP. 该实验的远 程客户使用 $512 \mathrm{Kbps}$ 连接速度的 modem 拨号连接. 利用 3 台计算机充当客户端: 一台是 Pentium 1.73 $\mathrm{GHz}$ 处理器, $512 \mathrm{MB}$ 内存, $64 \mathrm{MB}$ ATI 显卡的笔 记本电脑 (称之 PC-I). 第 2 台是 P4 $512 \mathrm{MB}$ 内存, GeForce2 显卡台式计算机 (称之 PC-II). 第 3 台是 P4 1 GB 内存, GeForce 3 显卡台式计算机 (称之 PCIII).

使用美国 Defense Mapping Agency 的 JGP95E 5 全球地形数据、USGS 的 GTOPO30 数据、 1:250000 全球地形数据、1:10000 荆江 DEM 数据, 全球影像为 $21600 \times 21600$ 的“blue marble”、30 m 的全国 TM 影 像、北京市区三维建筑模型库进行了实验研究.

基于 $\mathrm{COM}$ 组件技术, 用 $\mathrm{VC}++$ 和 OpenGL 开 发了运行在网络上的 3DGIS 可视化系统. 该系统由 三个模块构成: 第 1 个是基于 HTTP 的客户端模块, 负责从服务器上下载数据; 第 2 个是场景管理器模 块, 负责在数据储存器中加载数据, 并产生多分辨率 的三维场景; 第 3 个是三维分析模块, 用于地理空间 分析, 如最短路径分析、通视分析和水文分析等, 这 些模块相互联系用于 3DGIS 分析. 描述地形和三维 实体模型 (例如数据路径、范围、比例尺和配置) 的 元数据文件, 首先通过 HTTP 协议发送到客户端, 系 统利用文件中的该信息管理空间数据 ${ }^{[21]}$.

服务器根据场景元素的三角形和顶点的个数, 提供连续分辨率的描述, 而不是预先计算的若干个 细节层次模型. 在该实验中, 在服务器上计算荆江 $2048 \times 2048$ DEM 数据的简化误差需要 $1.92 \mathrm{~s}$, 计算 
北京朝阳区 2500 栋建筑模型的简化误差需要 $3.36 \mathrm{~s}$, 简化过程能够实时完成, 而且服务器为不需要每个客 户端渲染待可视化的模型, 所以本文的算法不会给服 务器造成很大负担.

表 8 列出可视化地形数据和三维实体模型的统 计信息, 可以看出数据从服务器端加载到客户端的 时间小于 $4 \mathrm{~s}$. 当客户端接到从服务器传来的响应时, 就产生数据的部分搭贝. 在客户端产生新请求之前, 客户端先对查询进行估算, 如果客户端未储存所需要 的结点, 则向服务器发出消息. 这样, 服务器就很容 易同时处理多个进程. 从图 6 和 7 中也能看出, 三维
场景的帧速率达到 32 帧/s, 每帧图像有 500000 个三 角形被渲染. 图 8 是应用程序部分可视化三维场景.

\section{5 结论}

本文提出了一种结点一层数据模型表达三维空 间数据的几何拓扑关系, 该数据模型根据客户端的请 求能够灵活的实现对大规模空间数据进行分割. 在 数据模型的基础上, 论述了基于网络体系的大规模 空间数据渲染方法. 借助于元素图、P-树空间索引、 视域裁剪和多线程技术实现了空间信息的渐进传输. 渐进传输算法根据数据类型把场景元素的信息分成

\section{表 8 数据可视化的统计信息}

\begin{tabular}{cccccccccc}
\hline 客户端 & $\begin{array}{c}\text { 原始数据 } \\
(\mathrm{kb})\end{array}$ & $\begin{array}{c}\text { 空间索引 } \\
(\mathrm{ms})\end{array}$ & $\begin{array}{c}\text { 压缩时间 } \\
(\mathrm{ms})\end{array}$ & $\begin{array}{c}\text { 视景体裁减 } \\
(\mathrm{ms})\end{array}$ & $\begin{array}{c}\text { 网络传输 } \\
(\mathrm{ms})\end{array}$ & $\begin{array}{c}\text { 解压数据 } \\
(\mathrm{ms})\end{array}$ & $\begin{array}{c}\text { 绘制三角形 } \\
(\mathrm{ms})\end{array}$ & $\begin{array}{c}\text { 渲染时间 } \\
(\mathrm{ms})\end{array}$ & $\begin{array}{c}\text { 绘制三角形 } \\
(\text { 个数 })\end{array}$ \\
\hline I & 4398 & 214 & 126 & 2 & 430 & 52 & 634 & 1458 & 218675 \\
II & 8128 & 356 & 278 & 2 & 781 & 102 & 858 & 2377 & 1026342 \\
III & 10375 & 425 & 346 & 3 & 852 & 219 & 1326 & 3171 & 3467425 \\
\hline
\end{tabular}

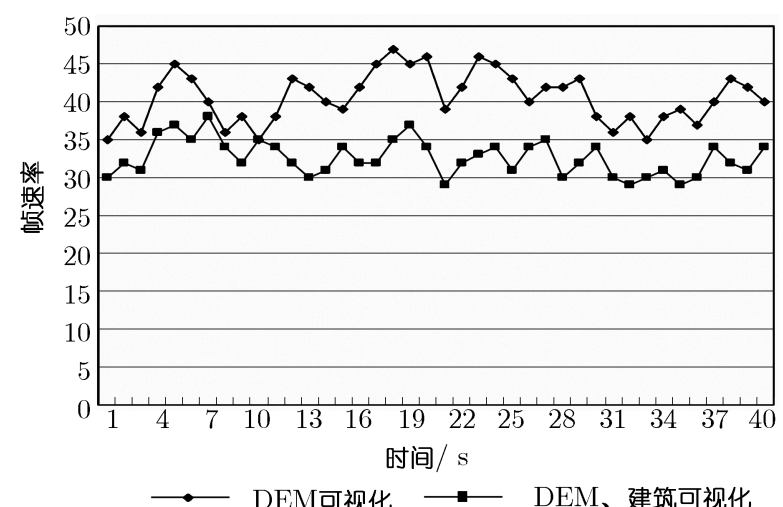

图 6 每秒绘制城市场景帧数的时间图

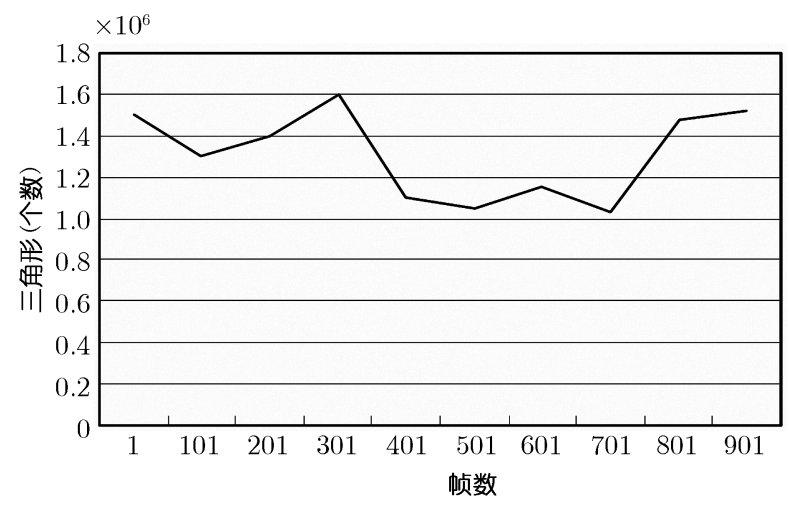

图 7 绘制三角形的时间一个数图 

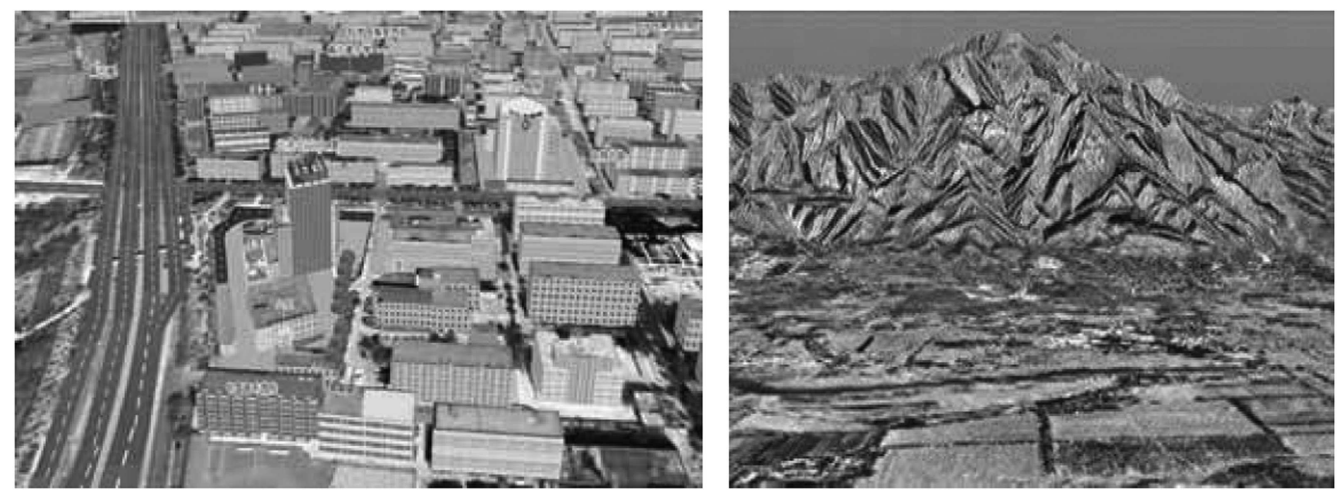

图 8 应用程序可视化的部分场景

几个数据流, 连续描述了服务器的三维场景, 根据客 户端的性能完成大数据自适应可视化. 与传统方法 相比, 本文的算法不仅可以极大缩短客户端的等待 时间, 而且降低了对网络传输速度、客户端和服务 器端硬件的配置要求. 但是这些算法的部分技术细 节还需要进一步完善和提高: 数据模型在构建复杂 不规则空间实体时, 由于现有表达和描述复杂空间 关系的不足, 会带来较大数据圥余; 再有, 服务器端 应用程序没有充分利用服务器本身的强大图形处理 能力.

数据传输和可视化过程中, 每一帧数据细化和简 化都需要顶点索引数组的更新, 但由于生成三角形条
带的函数调用过程是一致的, 相比起层次算法 ${ }^{[9]}$ 遍 历每一个顶点, CPU 重新计算索引的开销要小. 随 着 GPU 可编程性不断提高, 在新的图形硬件上利用 顶点纹理, 将索引的计算交给 GPU 来完成, 进而为 每一矩形块实时计算三角形条带索引数组, 通过图形 显卡的顶点缓冲和索引缓冲将会提高绘制速度. 在今 后工作中, 我们计划集成树结构模型与结点一层数据 模型, 来更好的表达复杂空间实体的拓扑关系和层次 多分辨率模型; 充分利用服务器的强大的计算和图形 处理能力, 增强其预处理能力和实时动态海量空间数 据的动态获取技术, 进一步加快大数据量数据传输速 度和可视化图形的质量.

\section{参考文献}

1 Losa A, Cervelle B. 3D Topological modeling and visualization for 3D GIS. Comput Graph, 1999, 23: 469-478

2 Gold C, Mostafavi M A. Towards the global GIS. ISPRS J Photogramm, 2000, 55(33): 150-163

3 Coors V. 3D GIS in networking environments. Comput Environ Urban Syst, 2003, 27(4): 345-357

4 龚健雅, 夏中国. 矢量与栅格集成的三维数据模型. 武汉测绘科技大学学报, 1997, 22(1): 7-15

5 李清泉, 李德仁. 三维空间数据模型集成的框架研究. 测绘学报, 1998, 27(4): 35-46

6 杨必胜. 数字城市的三维建模与可视化技术研究. 博士学位论文. 武汉: 武汉大学, 2002. 38-45

7 Teler E, Lischinski D. Streaming of complex 3D scenes for remote walkthroughs. Eurographics, 2001, 20(3): 125-132

8 Kim J, Lee S, Kobbelt L. View-dependent streaming of progressive meshes. In: 2004 International Conference on Shape Modeling and Applications (SMI 2004). Genova: Silver Spring MD: IEEE Computer Society, 2004. 209-220

9 Danovaro E, de Floriani L, Magillo P, et al. Level-of-detail for data analysis and exploration: a historical overview and some new perspectives. Comput Graph, 2006, 30(3): 334-344

10 El-Sana J, Sokolovsky N. View-dependent rendering for large polygonal models over networks. Int J Imag Graph, 2003, 3(2): $265-290$

11 Guthe M, Klein R. Streaming HLODs: an out-of-core viewer for network visualization of huge polygon models. Comput Graph, 2004, 28: 43-50

12 Zhang L Q, Zhang Y, Yang C J, et al. Effectve solutions to a global 3D visual system in networking environments. Sci China Ser D-Ear Sci, 2005, 48(11): 2032-2039

13 Rusinkiewicz S, Levoy M. Qsplat: a multiresolution point rendering system for lLarge meshes. In: Computer Graphics (Proceedings of Siggraph 2000). New York: ACM Press, 2000. 343-352 
14 Rusinkiewicz S, Levoy M. Streaming QSplat: a viewer for networked visualization of large, dense models. In: Symposium on Interactive 3D Graphics. Monterey: ACM Press, 2001. 63-68

15 Asirvatham A, Hoppe H. Terrain rendering using GPU-based geometry clipmaps. GPU Gems, 2005, 2: 27-44

16 Losasso F, Hoppe H. Geometry clipmaps: terrain rendering using nested regular grids. ACM Trans Graph, 2004, 23(3): $769-776$

17 Zhang L Q, Yang C J, Liu D L, et al. Visualization of large spatial data in networking environments. Comput Geosci-UK, 2007, 33(9): 1130-1139

18 Sahm J, Soetebier I, Birthelmer H. Efficient representation and streaming of 3D scenes. Comput Graph, 2004, 28(1): 15-24

19 Pouderoux J, Marvie J E. Adaptive streaming and rendering of large rerrains using strip masks. In: Proceedings of the 3rd International Conference on Computer Graphics and Interactive Techniques. Dunedin: IEEE Computer Society, 2005. 299-306

20 Cignoni P, Ganovelli F, Gobbetti E, et al. Planet-sized batched dynamic adaptive meshes (P-BDAM). In: IEEE Visualization'2003 Proceedings. Washington: IEEE Computer Seciety, 2003. 147-155

21 Zhang L Q, Yang C J, Liu D L, et al. A web-mapping system for real-time visualizing the global terrain. Comput Geosci-UK, 2005, 31(3): 445-454 